\title{
8 agosto 1944: Gropparello Liberata. Un progetto di public history alla vigilia del suo quinto compleanno
}

Un analisi del progetto 8 Agosto 1944. Gropparello Liberata a cinque anni dal suo avvio. Si tratta di un progetto che coniuga public history, microstoria e storia orale per celebrare la liberazione di Gropparello - piccolo comune dell'Appennino piacentino - da parte dei partigiani, avvenuta l'8 agosto 1944.

An analysis of the project 8 Agosto 1944. Gropparello Liberata started in 2012. The project connects public history, micro-history and oral history to celebrate the liberation of Gropparello - on small village in the Apennines near Piacenza- by the partisans, which occurred on August 8, 1944.

\section{Una comunità, una storia, un progetto}

Nelle zone appenniniche - dove la Resistenza nasce, si sviluppa, vive i suoi momenti di massima conflittualità - la memoria della lotta partigiana rimane una memoria diffusa, che si alimenta di racconti condivisi, ricordi individuali, episodi topici. A Piacenza, provincia ad alta densità partigiana, ogni comune, anche ogni frazione, ha i suoi eroi, i suoi luoghi di memoria, i suoi ricordi diffusi di battaglie vittoriose e sanguinose rappresaglie contro i civili. Si tratta di un patrimonio memoriale di inestimabile valore, che permette di gettare uno sguardo concreto sull'impatto che la guerriglia ha avuto nelle piccole comunità dell'Appennino - che più di tutte l'hanno sostenuta e vissuta sulla propria pelle - e sui modi in cui questa memoria è stata tramandata, collettivamente rielaborata, inserita nella 
stratificata identità locale.

Il recupero e la valorizzazione di episodi locali della Resistenza, dimenticati dalla storiografia nazionale ma ancora vivi nei racconti degli anziani e nelle tracce sul territorio, rappresenta allora un obiettivo primario per chi si occupa di public history nelle comunità locali più periferiche e lontane dal centro, ma anche per le pubbliche amministrazioni, che si trovano a gestire zone appenniniche sempre più spopolate, abitate da comunità sempre più anziane, esigue, disgregate e prive di punti di riferimento rispetto al passato e al futuro.

Da queste valutazioni nasce il progetto 8 agosto 1944. Gropparello Liberata promosso dal Comune di Gropparello (Piacenza) e coordinato dal Museo della Resistenza Piacentina di Morfasso (PC), istituzione nata nel 2009 che, oltre a rappresentare un luogo di memoria e meta di turismo in una zona di montagna priva di istituzioni culturali e centri di aggregazione, intende anche portare avanti progetti di promozione del territorio in chiave storica e momenti di approfondimento circa la storia contemporanea, dedicati alle comunità locali.

Al centro del progetto - partito nell'autunno del 2011 - sta la data dell' 8 agosto 1944, quando le formazioni partigiane locali, composte principalmente da giovani di Gropparello al comando di Primo Carini "Pip", scendono dal Monte Santa Franca - dove già dalla primavera si erano formati i primi nuclei di renitenti, ribelli e disertori. Obiettivo è la caserma di Via Marano, dove è asserragliato un contingente della GNR. Dopo alcune ore di assedio e combattimenti i partigiani del "Pip" riescono a costringere alla resa gli uomini della RSI. In paese si festeggia con canti e balli, mentre in Comune si insedia un'amministrazione civica, che fino ai rastrellamenti nazifascisti del gennaio 1945, guiderà Gropparello, in stretta collaborazione con le formazioni partigiane.

La scelta della data dell' 8 agosto 1944 risponde alla volontà di recuperare le memorie legate ad un episodio locale della Resistenza, che rischiava di perdersi con la fisiologica scomparsa dei testimoni. Un episodio in cui la comunità di Gropparello è protagonista, ma che spesso viene oscurato dalla festività del 25 aprile, unico momento condiviso di riflessione collettiva

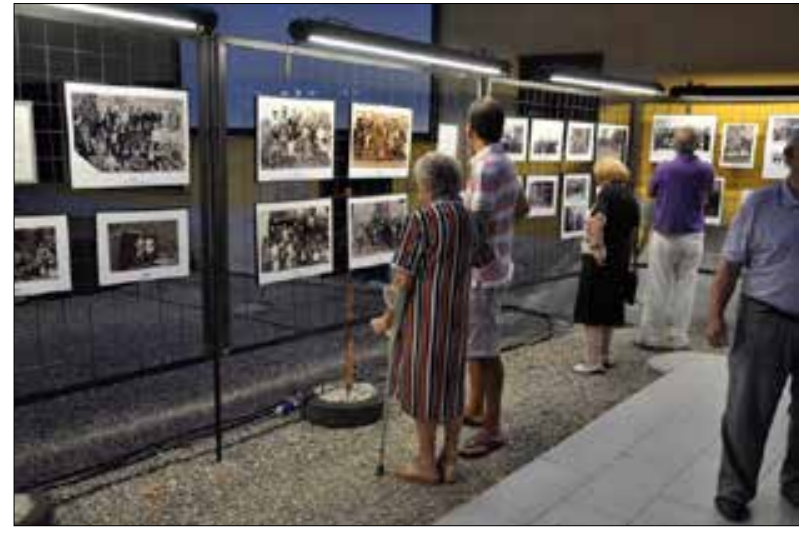

Gropparello, 8 agosto 2012: la mostra fotografica 
sulla Resistenza e sulla Liberazione. Forte, soprattutto da parte dell'Amministrazione Comunale, era la speranza che il progetto potesse avere anche una funzione sociale, recuperando legami di comunità e costruendo momenti di incontro e confronto tra generazioni.

Da qui l'idea di coinvolgere tutta la comunità di Gropparello nella raccolta e nella messa a sistema delle tracce sparse della zona libera partigiana nata l'8 agosto 1944, e di costruire precisi spazi di confronto e promozione del progetto che mettessero al centro la comunità locale, con le sue narrazioni, i suoi personaggi e luoghi storici, i suoi modi di ricordare la storia della Resistenza.

\section{II progetto: tra microstoria, digital public history, storia orale e ricerca diffusa}

La sfida del progetto, che ha l'ambizioso obiettivo di dare il via - attraverso il recupero della storia della Resistenza e della zona libera di Gropparello - ad un rilancio complessivo del sistema-paese, anche in chiave turistica, viene raccolto dagli operatori del Museo della Resistenza Piacentina, in particolare da Alessandro Pigazzini, coordinatore del progetto.

Il primo passo è la ricerca di coinvolgimento della comunità locale, anche grazie all'interessamento della sezione Anpi. Si procede così alla formazione di un "Gruppo di ricerca storica" composto da volontari del paese, con i quali si intende avviare una raccolta e sistematizzazione delle fonti relative alla zona libera formatasi l'8 agosto, e più in generale alla storia della Resistenza gropparellese, e al contesto socio-economico in cui si sviluppa.

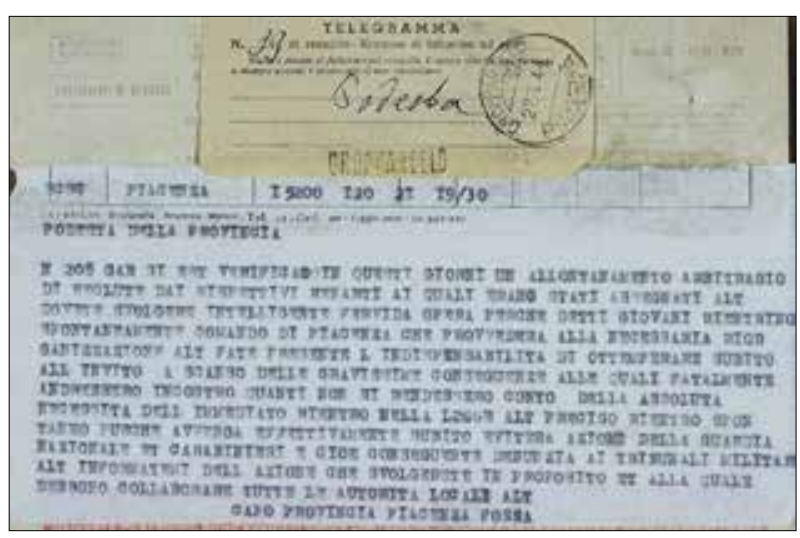

Telegramma del Capo della provincia al Podestà di Gropparello circa «i militari allontanatisi arbitrariamente dopo l's settembre», 22 gennaio 1944 (Archivio Comune di Gropparello, Collezione Gruppo di ricerca storica - 8 agosto 1944)
Il gruppo di volontari è stato coinvolto in un percorso di formazione della durata di tre mesi, curato dal personale del Museo, teso a restituire la complessità del periodo storico e a fornire alcuni elementi di base legati alla natura delle diverse fonti (fotografiche, documentali, orali), alla loro analisi e catalogazione attraverso schede standardizzate. 
I volontari del gruppo di ricerca hanno così potuto muoversi negli Archivi storici - quello del Comune di Gropparello in primis, ma anche l'Archivio di Stato di Piacenza, l'Archivio del Comitato Provinciale ANPI di Piacenza, l'Archivio Storico della Camera Provinciale di Commercio - fotografando, catalogando e analizzando in base a parole chiave e letture tematiche circa 12.000 documenti di diversa natura.

Si tratta di un materiale preziosissimo per gli studiosi, che ha permesso di ricostruire in modo agevole e con saldi appigli documentali, le ricadute pratiche dello squadrismo, dell'affermarsi del Regime, dell'entrata in guerra e della Resistenza sul territorio comunale. Nonostante il focus del lavoro rimanessero i mesi dell'Amministrazione civica infatti, $i$ volontari del gruppo di ricerca sono stati sensibilizzati circa la necessità

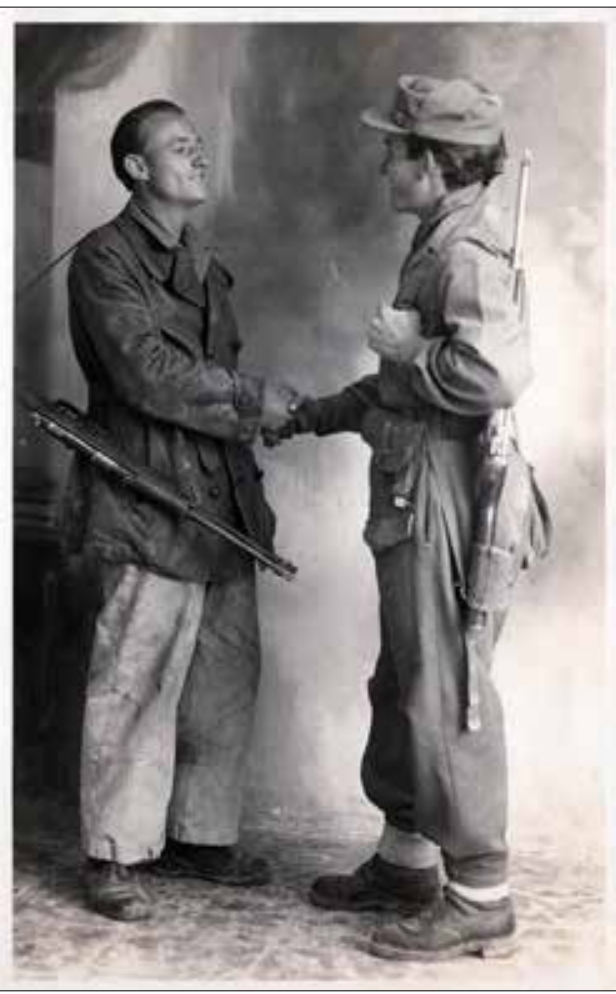

Ritratto fotografico di due partigiani di Gropparello: si riconosce Ugo Balduzzi "Tom" (foto gentilmente concessa dalla famiglia Baldini per la Collezione Gruppo di ricerca storica - 8 agosto 1944). di inserire questa esperienza di zona libera nel più ampio contesto del secondo conflitto mondiale e dell'occupazione nazifascista.

Altrettanto stimolante e utile è stato il lavoro di ricerca "porta a porta" nel corso del quale i volontari hanno contattato e coinvolto i loro compaesani, raccogliendo decine di testimonianze orali videofilmate, documenti e centinaia di fotografie messe a disposizione dai cittadini.

Altro soggetto che si è inteso coinvolgere nel lavoro di ricerca, analisi e archiviazione del materiale è la scuola secondaria di primo grado di Gropparello, per la quale gli operatori del Museo della Resistenza ha voluto elaborare progetti didattici ad hoc che sapessero inserirsi proficuamente nella quotidiana attività didattica, collegandosi con il programma di storia, e valorizzando l'attività dei ragazzi all'interno del progetto complessivo. Si è così creata una collaborazione stretta tra la scuola, gli insegnanti, e gli operatori del progetto, che ogni anno 
hanno coinvolto gli studenti un lavoro di ricerca finalizzato all'approfondimento di un aspetto specifico della lotta di Liberazione nel territorio di Gropparello, accompagnata da attività volte ad una comprensione più generale e complessiva del fenomeno resistenziale.

Pensato come un percorso triennale, il progetto didattico accompagna i ragazzi dal primo anno della scuola secondaria di primo grado - nel corso del quale viene loro proposto il laboratorio di animazione teatrale "Le nostre strade" - al terzo anno nel corso del quale i ragazzi sono accompagnati nella produzione di un prodotto storico, in grado di comunicare ai genitori e alla cittadinanza i risultati della loro "piccola"

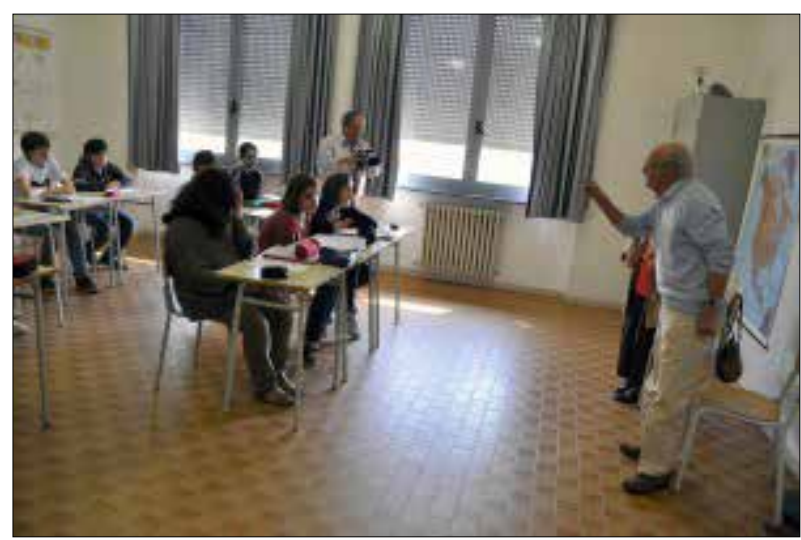

Ugo Magnaschi, partigiano della 142ma Brigata di manovra "E. Gallinari" operante a Gropparello, risponde alle domande dei ragazzi della 3 A dell'anno scolastico 2011-12). ricerca.

Un approfondimento particolare merita "Le nostre strade", primo suggestivo step del progetto didattico triennale, che intende coniugare dimensione ludico-ricreativa e dimensione formativo-didattica dell'apprendimento, e anche valorizzare il materiale e le testimonianze raccolte dai volontari gruppo di ricerca. Nato dalla collaborazione di storici, operatori museali ed esperti di historical reenactment, il laboratorio coinvolge i ragazzi in una narrazione che unisce i racconti dei testimoni - presenti in sala e disponibili a confrontarsi e dialogare con gli studenti al termine del laboratorio - la musica dal vivo, e particolari momenti di riflessione autonoma e immedesimazione dei giovani spettatori.

In questi anni il rapporto con l'istituzione scolastica e con i docenti si è rivelato di fondamentale importanza per il coinvolgimento della cittadinanza e per incentivare il dialogo tra diverse generazioni, ed ha prodotto diversi materiali messi a disposizione del gruppo di ricerca, come l'opuscolo Accadeva a Montechino, nel quale i ragazzi hanno ricostruito - grazie all'analisi fonti d'archivio, fonti materiali e fonti orali - la storia della frazione gropparellese di Montechino, sede di un'importante giacimento petrolifero, o il video Generazioni a Confronto (vincitore del Premio "Filmare la Storia" dell'Archivio nazionale cinematografico della Resistenza, edizione 2014), che racconta il dialogo tra i ragazzi e gli anziani del 
paese, che si sono resi disponibili ad incontrare gli studenti e rispondere alle loro domande sulla Resistenza e sulla vita quotidiana di quegli anni.

\section{Una comunità libera, che sa anche cantare e ballare}

Parallelamente all'attività di ricerca storica "plurale", e al lavoro didattico nelle scuole, il progetto "8 agosto 1944. Gropparello Liberata" si compone di un altro momento fondamentale: l'evento che ogni anno viene organizzato proprio nella ricorrenza dell' 8 agosto, che a partire dal 2012 ha animato Gropparello, con incontri di studio, spettacoli artistici, concerti, e mostre legate alla Resistenza. Si tratta di un'attività di promozione, comunicazione, coinvolgimento della popo-

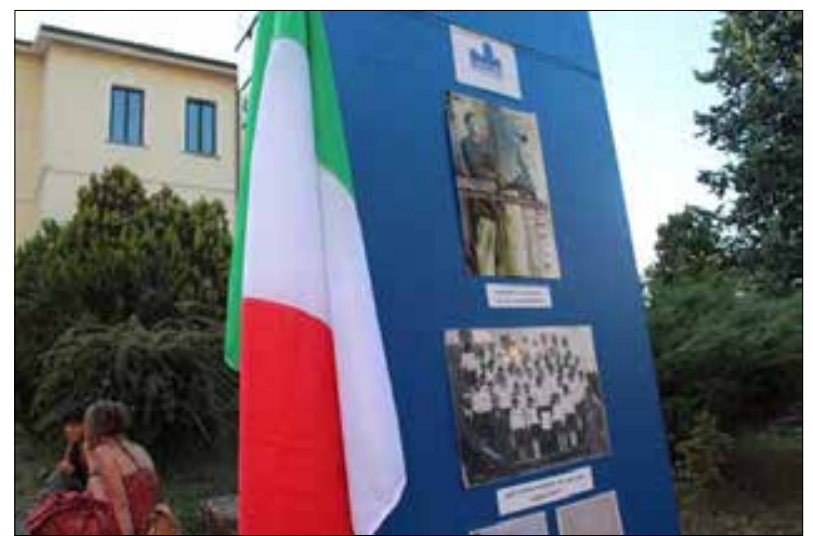

Uno dei totem sparsi per Gropparello a formare una mostra fotografica diffusa.

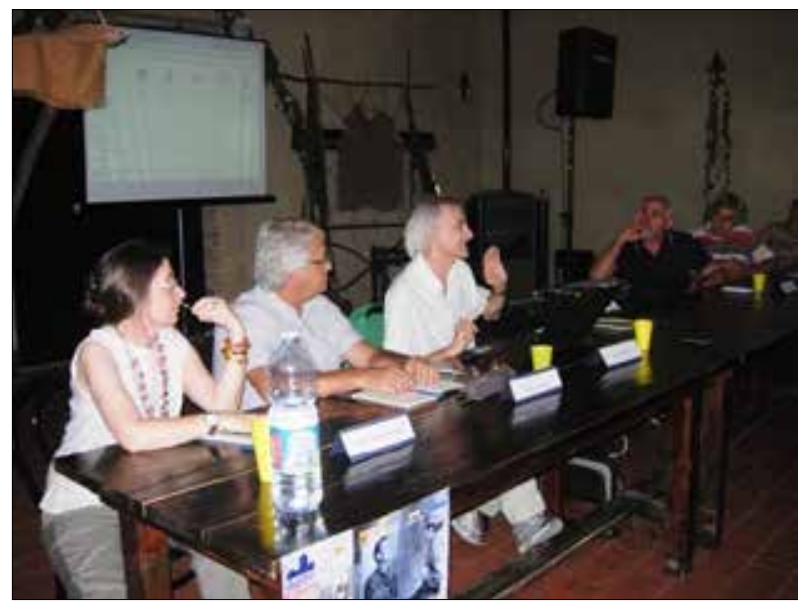

Uno dei totem sparsi per Gropparello a formare una mostra fotografica diffusa. lazione e dei gruppi operanti sul territorio, che intende sia "fissare" nella memoria comunitaria la data periodizzante della prima liberazione del paese, sia comunicare in modo accessibile i risultati delle attività didattiche e di ricerca.

Attraverso imponenti "totem", le fotografie reperite dai volontari del gruppo di ricerca vengono affisse per le strade di Gropparello, rendendo visibili ai passanti archivi fotografici famigliari che altrimenti sarebbero rimasti sepolti nei cassetti. In collaborazione con 1'Istituto Storico della Resistenza e dell'età contemporanea di Piacenza viene organizzata una conferenza su un tema di interesse storico, cercando di far 
confluire a Gropparello, paese appenninico lontano da università e centri di ricerca, storici di rilevanza nazionale.

L'unione della ricostruzione storica con l'attività sportivo-escursionistica è alla base della passeggiata storico-naturalistica "Sui sentieri del Pip", che ogni anno accompagna giovani, bambini e famiglie in una passeggiata sui luoghi della Resistenza a Gropparello, con l'emozionante racconto dei fatti accaduti, ricostruiti grazie al materiale raccolto dal gruppo di ricerca. Si tratta di una forma particolare di raccontare la storia locale, che negli anni ha visto crescere l'interesse e la partecipazione.

Alla sera il progetto 8 agosto 1944: Gropparello Liberata entra nella sua parte più conviviale: in un grande evento conclusivo si raccontano i risultati delle attività di ricerca e divulgazione, si mangia, si ascolta musica e si balla. Un aspetto che appare secondario ma che è risultato centrale nel coinvolgimento della comunità - che dal 2012 ha visto confluire in paese persone interessate alla storia ma anche semplici curiosi da tutta la provincia. Con il contributo del Comune di Gropparello e della locale sezione Anpi si sono potuti organizzare, anno dopo anno, spettacoli di vario tipo, dal teatro, alla musica rock, al canto tradizionale e corale, tutti legati alla storia della Resistenza e ai suoi personaggi. Uno step, quello dell'entertainment, che si è rivelato progressivamente di fondamentale importanza, riuscendo a coinvolgere e divertire fasce diverse di pubblico, far collaborare diversi individui ed associazioni locali nei preparativi per quella che ormai tutti a Gropparello chiamano «la festa partigiana dell'8 agosto» e valorizzare agli occhi dei compaesani e della Provincia tutto il lavoro di ricerca, didattica e divulgazione.

\section{Verso l'8 agosto 2016...}

Sarà proprio l'evento previsto per il prossimo 8 agosto a chiudere definitivamente il progetto, con la presentazione del volume che raccoglie il lavoro svolto dal gruppo di ricerca, dalla scuola e dai ricercatori coinvolti a partire dal 2012, al quale seguiranno i canti della conferenza-spettacolo sulla memoria della Resistenza nella musica italiana ...e questo è il fiore del partigiano.

Sarà anche il momento per valutare a fondo i risultati finali di questa complessa iniziativa, che però appaiono già buoni. Da una prima raccolta di feedback dei diversi attori compiuta da Iara Meloni nel suo volume Memorie Resistenti [Le Piccole Pagine, 2015], il progetto 8 agosto 1944. Gropparello Liberata appare come un'esperienza coinvolgente e in grado di attivare e rivitalizzare legami comunitari. Una volontaria del gruppo di ricerca storica ad esempio dichiara: Il progetto "8 agosto 1944" è stata per me un'esperienza molto intensa e impor- 
tante. Mi ha permesso, oltre che di approfondire la storia del territorio, di conoscere meglio molte persone e scambiare con loro l'esperienza di una ricerca di gruppo finalizzata a "conservare e tramandare" la storia di questo paese. [S.C. in Meloni, 2015: 199].

Un entusiasmo condiviso dagli studiosi e dagli operatori coinvolti che, nel corso di una serie di recenti focus group, hanno individuato nel progetto 8 agosto 1944 un proficuo esempio di storia fatta nelle e con le comunità locali, nonché un'occasione per conoscersi tra soggetti diversi, che pur vivendo in ambienti sociali separati possono, attraverso le pratiche di public history, riconoscersi intorno a una narrazione storica condivisa. Il tal modo, è stato possibile sottrare le celebrazioni della Resistenza a una retorica ingessata e incapace di instaurare un reale dialogo con le comunità.

\section{Risorse on line}

Il progetto sul web e sui social

http://www.8agosto1944.it/

https://www.youtube.com/user/8agosto1944

https://www.facebook.com/otto.agosto.3

Frammento di videointervista: la staffetta partigiana "Mina" Callegari racconta una missione https:/www.youtube.com/watch?v=qRuPlSgrSqs

Podcast del convegno L'estate partigiana http://www.casamemoriavinchio.it/index.php?option=com_content\&view=article\&id =25: convegno-1943-1945-guerra-rappresaglie-territori\&catid=21\&Itemid= 131 